\title{
Theory of the Bloch Oscillating Transistor
}

\author{
J. Hassel, and H. Seppä \\ VTT Information Technology, Microsensing, P.O. Box 1207, FIN-02044 VTT
}

\begin{abstract}
The Bloch oscillating transistor (BOT) is a device, where single electron current through a normal tunnel junction can be used to enhance Cooper pair current in a mesoscopic Josephson junction leading to signal amplification. In this paper we develop a theory, where the BOT dynamics is described as a two-level system. The theory is used to predict current-voltage characteristics and small-signal response. Transition from stable operation into hysteretic regime is studied. By identifying the two-level switching noise as the main source of fluctuations, the expressions for equivalent noise sources and the noise temperature are derived. The validity of the model is tested by comparing the results with simulations.
\end{abstract}

PACS numbers: 74.78.Na, 85.25.Am, 85.35.Gv

\section{INTRODUCTION}

The Bloch oscillating transistor $(\mathrm{BOT})^{\underline{1}-\underline{\underline{5}}}$ is a device based on tuning the probability of interlevel switching in a mesoscopic Josephson Junction (JJ). The equivalent circuit is shown in Fig. 1(a). The current $I_{C}$ at the collector $(\mathrm{C})$-emitter(E) -circuit is controlled by the base current $I_{B}$ leading to transistor-like operation. The physics behind this is based on controlling the state of the JJ by means of quasiparticles tunneling through the normal tunnel junction connected to the base electrode (B).
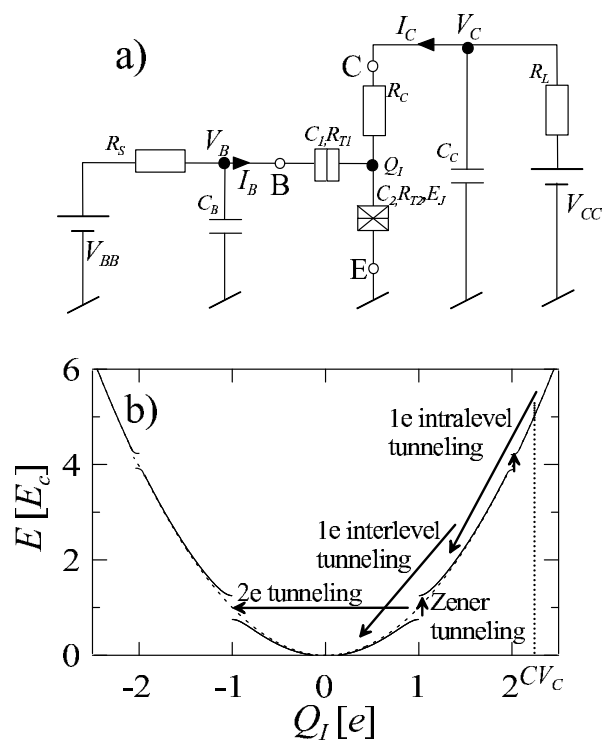

FIG. 1: (a) Schematic circuit of a BOT connected to a source and a load. Source $R_{S}$ is connected to the base electrode B and load $R_{L}$ to the collector electrode C. The lead capacitances from the electrodes to the ground are $C_{B}$ and $C_{C}$. The BOT itself consists of a Josephson Junction (JJ) between the emitter electrode E and the superconducting "island", normal tunnel junction between $\mathrm{B}$ and the island and large $R \gg R_{Q}$ resistor between $\mathrm{C}$ and the island. (b) The state diagram of the JJ and possible transitions induced by charge tunneling through the junctions.
The state diagram as function of the "island" (quasi)charge $Q_{I}$ is shown in Fig. $1(\mathrm{~b}) \underline{\underline{\underline{6}}}$, where also the transitions are illustrated. It is assumed that the Josephson coupling energy $E_{J}$ is smaller or of the same order as the charging energy $E_{C}=e^{2} / 2 C_{\Sigma}$, and that $R, R_{T 1}, R_{T 2} \gtrsim R_{Q}$. Here $C_{\Sigma}$ is the total capacitance of the island, $R$ is the collector resistor, and $R_{T 1}$ and $R_{T 2}$ are the tunnel resistances of the two junctions. The quantum resistance $R_{Q}=h / 4 e^{2} \approx 6.5 \mathrm{k} \Omega$. When $\mathrm{C}$ is biased at a point, where $V_{C} \gtrsim e / C_{\Sigma}$, the charge tends to relax through the collector resistor $R_{C}$ towards $V_{C} C_{\Sigma}$. Here $V_{C}$ is the collector voltage. At $Q_{I}=e$ it is likely that a Cooper pair (CP) tunneling through the JJ returns the island back to $Q_{I}=-e$. Repeating this cycle, the Bloch Oscillation ${ }^{6}$, leads to a net current through the CE-circuit. A competing process is the Zener tunneling? which takes the system to the upper bands $\left(\left|Q_{I}\right|>e\right.$ in the extended band picture we are using). There the Cooper pair tunneling is blocked. One or more quasiparticles tunneling through the base junction may return the system back to the lowest energy band i.e. back to $\left|Q_{I}\right|<e$.

The Zener tunneling probability between bands $n$ and $n-1$ is given as $P_{n-1 \rightleftharpoons n}^{Z} \approx$ $\exp \left(-(\pi / 8) / n^{2 n-1}\left(E_{J} / E_{C}\right)^{2 n}\left(e E_{C} / \hbar I_{0}\right)\right)$. In the limit of small $E_{J}$ it approaches unity very rapidly as $n$ increases. Therefore we can neglect Cooper pair tunneling at higher bands, and consider the device as a two-level system. Below we will call the lowest band with $n=0$ the "first level" and higher bands with $n \gtrsim 1$ the "second level". Tunneling events, which cause transitions between the levels will be called interlevel transitions (or upwards and downwards transitions) and those, which cause transitions between higher bands, will be called intralevel transitions. Controlling the probability of downwards transitions leads to control of average $I_{C}$ and thus to transistor-like characteristics.

The BOT was recently experimentally realized ${ }^{3}$, and simulations showed that its properties can be quantitatively predicted with a computational model ${ }^{5}$. It is potentially useful in cryogenic applications such as readout 
circuits of radiation detectors, or measurement of small currents in quantum metrology. The aim of this article is to gain more insight into the dynamics of the BOT and to find the noise properties. To be able to do so, we derive an analytic theory, and study its applicability by comparing the results to computational data. Small-signal parameters of BOT as well as equivalent noise sources and the noise temperature are derived.

\section{COMPUTATIONAL MODEL}

Since we assume that $E_{J} \ll E_{C}$ we can interpret the quasicharge as the real charge, and the energy diagram will reduce to the simple parabola (dashed line in Fig. 1b). We can account for the Josephson coupling perturbatively and calculate the $\mathrm{CP}$ tunneling probabilities using the $P(E)$-theory ${ }^{8.9}$. This takes the electromagnetic environment into account. The consequence is that the tunneling does not happen strictly at $Q_{I}=e$, but is rather represented with a finite distribution ${ }^{5}$.

The simulation is done by integrating $Q_{I}$ in the time domain. The charge $Q_{I}=Q_{2}-Q_{1}$ becomes

$\frac{d Q_{I}}{d t}=\frac{V_{C}-V_{2}}{R_{c}}-\left(\frac{d Q_{I}}{d t}\right)_{Q P 1}-\left(\frac{d Q_{I}}{d t}\right)_{Q P 2}-\left(\frac{d Q_{I}}{d t}\right)_{C P}$,

where the first term is the collector current, the second and the third terms describe quasiparticle tunneling through the two junctions and the last term describes the Cooper pair tunneling through the Josephson junction. The corresponding tunneling rates as the function of the state of the system are derived below. The voltage $V_{2}$ is across the JJ. The charges and voltages across both junctions, $Q_{1}$ (base junction) and $Q_{2}$ (JJ) needed for the tunneling rate computation below are obtained as follows:

$$
\begin{aligned}
& Q_{1}=\frac{1}{C_{\Sigma}}\left(C_{1} C_{2} V_{B}-C_{1} Q_{I}\right) \\
& Q_{2}=\frac{1}{C_{\Sigma}}\left(C_{1} C_{2} V_{B}+C_{2} Q_{I}\right)
\end{aligned}
$$

The voltages across the junctions are $V_{i}=Q_{i} / C_{i}$. In numerical simulations we will assume zero load resistance $\left(R_{L}=0\right)$, i.e. the collector voltage $V_{C}=V_{C C}$ is fixed. In some simulations the base electrode is assumed voltage biased (i.e. $R_{S}=0$ and $V_{B}=V_{B B}$ ). Note that below we will also use parameter $V_{B}^{\prime}=V_{B}-V_{C}$ as base bias parameter for convenience. From the circuit point of view the choice between $V_{B}$ and $V_{B}^{\prime}$ is the detailed bias arrangement.

In some simulations $R_{S}$ will be assumed much higher than the input impedance. This fixes the base current
$I_{B}$. In this case $V_{B}$ is integrated as

$$
\frac{d V_{B}}{d t}=\frac{1}{C_{B}}\left(I_{B}+\left(\frac{d Q_{I}}{d t}\right)_{Q P 1}\right),
$$

where $I_{B}$ is the base current and $C_{B}$ is the cable capacitance. Here we have assumed that $C_{B} \gg C_{\Sigma}$.

Both Cooper pair and quasiparticle tunneling probabilities are obtained from the $P(E)$-theory ${ }^{9}$, so that the effect of electromagnetic environment (the collector resistor) is also included. The given tunneling rates are towards the island. By reversing the signs of voltages $V_{1}$ and $V_{2}$ the rates from the island are obtained. The Cooper pair tunneling rate is

$$
\Gamma_{C P}\left(V_{2}\right)=\frac{\pi E_{J}^{2}}{2 \hbar} P\left(2 e V_{2}\right),
$$

and $P(E)$ is defined as

$$
P(E)=\frac{1}{2 \pi \hbar} \int_{-\infty}^{\infty} d t \exp \left(J(t)+\frac{i}{\hbar} E t\right),
$$

where the phase correlation function $J(t)$ is

$$
\begin{aligned}
J(t) & =2 \int_{0}^{\infty} \frac{d \omega}{\omega} \frac{R_{C} / R_{Q i}}{1+\left(\omega R_{C} C_{\Sigma}\right)^{2}} \\
& \times\left[\operatorname{coth}\left(\frac{1}{2} \frac{\hbar \omega}{k_{B} T}\right)(\cos (\omega t)-1)-i \sin (\omega t)\right]
\end{aligned}
$$

Here $R_{Q 2}=h / 4 e^{2} \approx 6.5 \mathrm{k} \Omega$ is the quantum resistance for Cooper pairs. For the quasiparticle tunneling formulas given below value $R_{Q 1}=h / e^{2} \approx 25.9 \mathrm{k} \Omega$ is used instead. Capacitance $C_{\Sigma}=C_{1}+C_{2}$ is the total capacitance of both junctions, $T$ is the temperature and $k_{B}$ the Bolzmann constant.

The quasiparticle tunneling rates through the junctions $(i=1$ for the base junction and $i=2$ for the JJ) are

$$
\begin{aligned}
\Gamma_{Q P i}\left(V_{i}\right) & =\frac{1}{e^{2} R_{T i}} \int_{-\infty}^{\infty} \int_{-\infty}^{\infty} d E d E^{\prime} \frac{N_{1}(E)}{N_{1}(0)} \frac{N_{2}\left(E^{\prime}+e V_{i}\right)}{N_{2}(0)} \\
& \times f(E)\left[1-f\left(E^{\prime}+e V_{i}\right)\right] P\left(E-E^{\prime}\right)
\end{aligned}
$$

where $R_{T i}$ are the tunneling resistances. The densities of states on both sides of the junctions are $N_{1}(E)$ and $N_{2}(E)$. For both NIS junction and the JJ $N_{1}(E) / N_{1}(0)=\Theta\left(E^{2}-\Delta^{2}\right) E / \sqrt{E^{2}-\Delta^{2}}$. For the JJ $N_{2}(E) / N_{2}(0)=\Theta\left(E^{2}-\Delta^{2}\right) E / \sqrt{E^{2}-\Delta^{2}}$ and for the NIS Junction $N_{2}(E) / N_{2}(0)=1$. The superconducting 
gap is $\Delta, f(E)$ is the Fermi function and $\Theta(E)$ is the step function.

One should notice that the voltages appearing in the tunnel rate formulas are time dependent as opposed to the standard $P(E)$-theory, where the fixed bias voltage is used. By doing so we can account for the fact that the tunneling probabilities depend on the state of the system.

\section{ANALYTIC THEORY}

In the theory derived below, BOT is modelled as a mapping of voltages $V_{B}$ and $V_{C}$ into currents $I_{C}$ and $I_{B}$. We assume that a single tunneling event will not affect the voltages. This is the case, since $C_{B}, C_{C} \gg C_{\Sigma}$ in a practical experimental setup.

We assume that $1 \ll E_{C} / k T \ll R_{C} / R_{Q}$ and $E_{J} \ll$ $E_{C}$, which means that the Cooper pair tunneling rate (Eq. (5)) reduces to a delta spike centered at $\left|Q_{I}\right|=\epsilon^{5}$. This recovers our interpretation of the two-level system with $\left|Q_{I}\right|<e$ as the first level and $\left|Q_{I}\right|>e$ as the second level. We also assume that $C_{2} \gg C_{1}$ and neglect quasiparticle tunneling through the JJ. Below unnecessary subscripts for capacitances and charges are dropped, i.e. $C \equiv C_{2} \equiv C_{\Sigma}, R_{T} \equiv R_{T 1}$ and $Q \equiv Q_{I} \equiv Q_{2}$. We analyze only the regime, where $V_{C}>e / C$ and $V_{B}^{\prime}<0$, since this is interesting for the amplifier operation.

The collector current is written as

$$
I_{C}=\frac{1 / \Gamma_{\uparrow}}{1 / \Gamma_{\uparrow}+1 / \Gamma_{\downarrow}} I_{S}-I_{B}
$$

where the first term is the Cooper pair current through the Josephson junction and $I_{B}$ is the single electron current through the base electrode. The transition rates between the two levels are $\Gamma_{\uparrow}$ and $\Gamma_{\downarrow}$. The "saturation current", i.e. current through the JJ at the first level, is $I_{S}=2 e f_{B}$, where $f_{B}$ is the Bloch oscillations frequency.

The base current is

$$
I_{B}=-\frac{\left\langle N_{e}\right\rangle e}{1 / \Gamma_{\uparrow}+1 / \Gamma_{\downarrow}}
$$

where $\left\langle N_{e}\right\rangle$ is the number of electrons needed to induce a downwards transition. Here we have neglected the possibility of single-electron tunneling, when the system is at the first level. This is justified, since typically the voltage $\left|V_{1}\right|$ is below the gap voltage in that case. The Eqs. (9) and (10) give general IV characteristics for the BOT.

Between tunneling events $d Q / d t=\left(V_{C}-Q / C\right) / R$. By integrating from $Q=-e$ to $Q=e$, i.e. over one Bloch period one gets

$$
f_{B}=\frac{1}{R C}\left[\ln \left(\frac{V_{C} / V_{Q}+1}{V_{C} / V_{Q}-1}\right)\right]^{-1}
$$

or

$$
I_{S}=\frac{2 e}{R C}\left[\ln \left(\frac{V_{C} / V_{Q}+1}{V_{C} / V_{Q}-1}\right)\right]^{-1},
$$

where we have defined $V_{Q}=e / C$.

The upwards tunneling rate (the Zener tunneling) can now be written as ${ }^{10}$

$$
\Gamma_{\uparrow}=\frac{I_{S}}{2 e\langle N\rangle},
$$

where

$$
\langle N\rangle=\exp \left(\frac{I_{z}}{e} \frac{R C}{V_{C} / V_{Q}-1}\right)-1
$$

is the average number of Cooper pairs in one sequence of Bloch oscillations. One sequence here means the time between tunneling down to the first level and tunneling back to the second level. The Zener avalanche current is $I_{z}=\pi e E_{J}^{2} / 8 \hbar E_{c}$.

The downwards tunneling at low temperatures and for large $R$ is exclusively due to single electron tunneling through the base junction. To calculate $\left\langle N_{e}\right\rangle$ and $\Gamma_{\downarrow}$ we first derive an approximation for $\Gamma_{Q P 1}$. The most general form is obtained from Eq. (8). For many purposes, however, a piecewise linear approximation is sufficient:

$$
\begin{aligned}
\Gamma_{Q P 1}(t) & =\frac{1}{R_{T} C}\left(\frac{V_{1}}{V_{Q}}-\frac{1}{2}\right) \\
& =\frac{1}{R_{T} C}\left(\frac{Q(t)}{e}-\frac{V_{C}+V_{B}^{\prime}}{V_{Q}}-\frac{1}{2}\right), \text { if }\left|V_{1}\right|>V_{G}
\end{aligned}
$$

$\Gamma_{Q P 1}(t)=0$, if $\left|V_{1}\right|<V_{G}$,

where the gap-voltage is $V_{G}$, i.e., we neglect the leakage current at the subgap voltages. In the most straightforward experimental realization the base junction is a NIS junction. In this case $V_{G}=\Delta+V_{Q} / 2$ including the contribution of both superconducting and Coulomb gaps. In principle it is also possible to realize the base junction as a NIN junction, i.e. with suppressed superconductivity on the other electrode. Then the gap voltage is $V_{G}=V_{Q} / 2$. In general, $\Gamma_{Q P 1}$ is a function of time due to the time dependency of charge.

We now have to separate two different regimes to find analytic approximations for $\left\langle N_{e}\right\rangle$ and $\Gamma_{\downarrow}$. If $V_{C}<2 V_{Q}$ one electron always suffices to return the system to the first level, i.e., $\left\langle N_{e}\right\rangle=1$. In this case the probability distribution of the first quasiparticle tunneling event after the Zener tunneling is

$$
P(t)=\Gamma_{Q P 1}(t) \lim _{\Delta t \rightarrow 0} \prod_{j=1}^{t / \Delta t}\left(1-\Gamma_{Q P 1}(j \Delta t)\right)
$$


which is the probability that an electron will tunnel at time $t$ times the probability that it has not tunneled at earlier times. The charge in Eq. (15) before the first quasiparticle event obeys simple $R C$-relaxation, i.e.

$$
Q(t)=C V_{C}\left(1+\left(\frac{V_{Q}}{V_{C}}-1\right) e^{-\frac{1}{R C} t}\right)
$$

where we assumed that Zener tunneling occurs at $t=0$ (or equivalently that $Q(0)=e$ ). The average rate $\Gamma_{\downarrow}$ is the inverse of the weight of the distribution given by Eq. (16). Thus

$$
\begin{aligned}
\left\langle N_{e}\right\rangle & =1 \\
\Gamma_{\downarrow} & =\frac{1}{\int_{0}^{\infty} t P(t) d t} .
\end{aligned}
$$

In general, the downwards rate has to be evaluated numerically from (19). However, if we further assume that the transient in Eq. (17) is short, Eq. (16) reduces to a simple exponential distribution. This is equivalent to assuming that $Q(t) \approx C V_{C}$ at all times, and thus Eq. (15) also becomes time independent. Now simply $\Gamma_{\downarrow}=\Gamma_{Q P 1}$, and it follows

$$
\Gamma_{\downarrow}=-\frac{1}{C R_{T}}\left(\frac{V_{B}^{\prime}}{V_{Q}}+\frac{1}{2}\right) .
$$

If $V_{C}>2 V_{Q}$ it is possible that the first electron tunneling through the base junction does not cause a transition to the first level, but some of the single quasiparticle events lead to intralevel transitions instead. This was found to have a dramatic effect in the experiments $\frac{3}{,}$, and is found to be an important issue from the device optimization point of view as well. To solve $\left\langle N_{e}\right\rangle$ and $\Gamma_{\downarrow}$ analytically from Eqs. (15) and (17) in this case is unfortunately impossible. To find a sufficient approximation for our purposes, we have solved the problem numerically and searched for a proper fitting function. For simplicity we have assumed an NIN junction at the base electrode, i.e. $V_{G}=V_{Q} / 2$ in Eq. (15). Some fits are shown in Fig. 2 and the result is

$$
\begin{aligned}
\left\langle N_{e}\right\rangle & =0.04\left(\frac{R_{T}}{R}\right)^{2} \\
& \times \exp \left(0.3 \exp \left(1.8 \frac{V_{C}}{V_{Q}}+0.27 \frac{V_{C} V_{B}^{\prime}}{V_{Q}^{2}}-0.2 \frac{V_{B}^{\prime}}{V_{Q}}\right)\right)+1 \\
\Gamma_{\downarrow}^{-1} & =1.2 e \frac{R+R_{T}}{V_{B}^{\prime}}\left(1-\left\langle N_{e}\right\rangle\right) \\
& +R C\left(2.5 \frac{R_{T}}{R}+1.1\right)\left(\frac{V_{Q}}{V_{B}^{\prime}}\right)^{2} .
\end{aligned}
$$

The fit is accurate, when $R_{T} \lesssim R$. The weaker dependence indicated by the unity term in Eq. (21) and $\left(2.5 R_{T} / R+1.1\right)\left(V_{B}^{\prime} / V_{Q}\right)^{2}$ term in Eq. (22) dominate at $V_{C} \approx 2 V_{Q}$ and large $\left|V_{B}^{\prime}\right|$. In this case only one quasiparticle is needed to induce a downwards transition. This is possible, if the tunneling occurs during the transient immediately after the Zener tunneling, while still $Q(t)<2 e$. The terms are actually an approximation of Eqs. (18) and (19) at $V_{C} \approx 2 V_{Q}$. The $\exp (0.3 \exp (\ldots))$-term dominates, when several tunneling events are needed to induce an interlevel transition. The very strong dependence is roughly explained as follows. Let us assume that $2 V_{Q}<V_{C}<3 V_{Q}$ and the island charge is initially $Q \approx C V_{C}$ (see Fig. 1). Now at least two quasiparticles tunneling rapidly one after another are needed to induce a downwards transition. The quasiparticle tunneling probability according to Eq. (15) is at its maximum, when $Q \approx C V_{C}$. However, after the first tunneling event $Q$ drops down to $C V_{C}-e$ and therefore the probability also drops. Hence the probability for the second quasiparticle to tunnel before the charge relaxes back to $Q>2 e$ is small. The charge therefore tends to oscillate between $Q \approx C V_{C}$ and $Q \approx C V_{C}-e$ for a long time before the rather improbable event at $Q<2 e$ happens. This generates a large quantity of intralevel transitions thus increases $\left\langle N_{e}\right\rangle$ and $\Gamma_{\downarrow}$.

\section{COMPARING NUMERIC AND ANALYTIC IV CURVES}

In Fig. 3(a) we show a simulated set of $I_{C}-V_{C}$ curves (open circles), where the base is voltage biased. The base voltage $V_{B}^{\prime}$ is varied, while other parameters are $R=1.5$
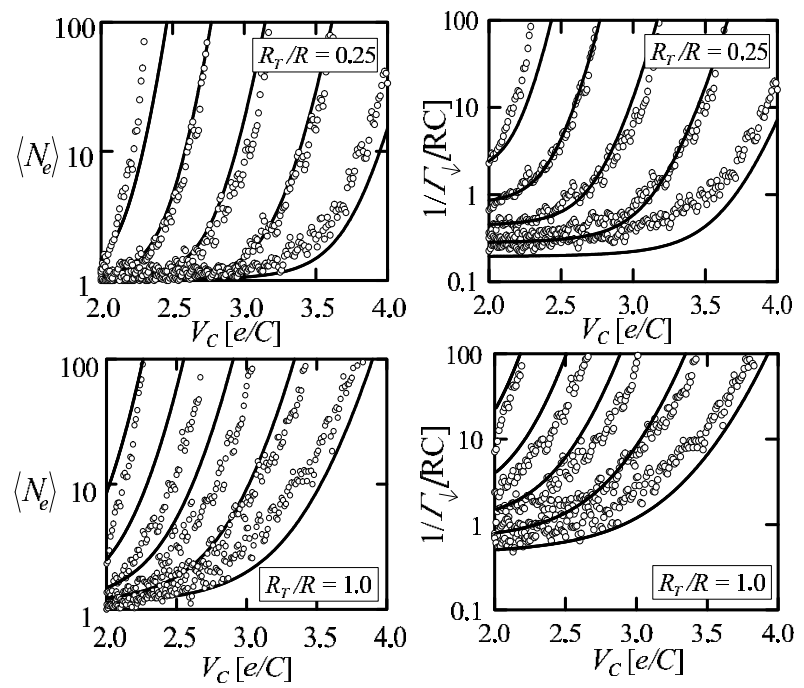

FIG. 2: Computed data for $\left\langle N_{e}\right\rangle$ and $\Gamma_{\downarrow}$ obtained by solving Eqs. (15) and (17) numerically (open circles). In each frame the base voltage is varied as $V_{B}^{\prime}=-1.0 e / C,-1.5 e / C,-2.0 e / C$, $-2.5 e / C,-3.0 e / C$ from left to right. The lines correspond the fits, i.e. Eqs. (21) and (22). 

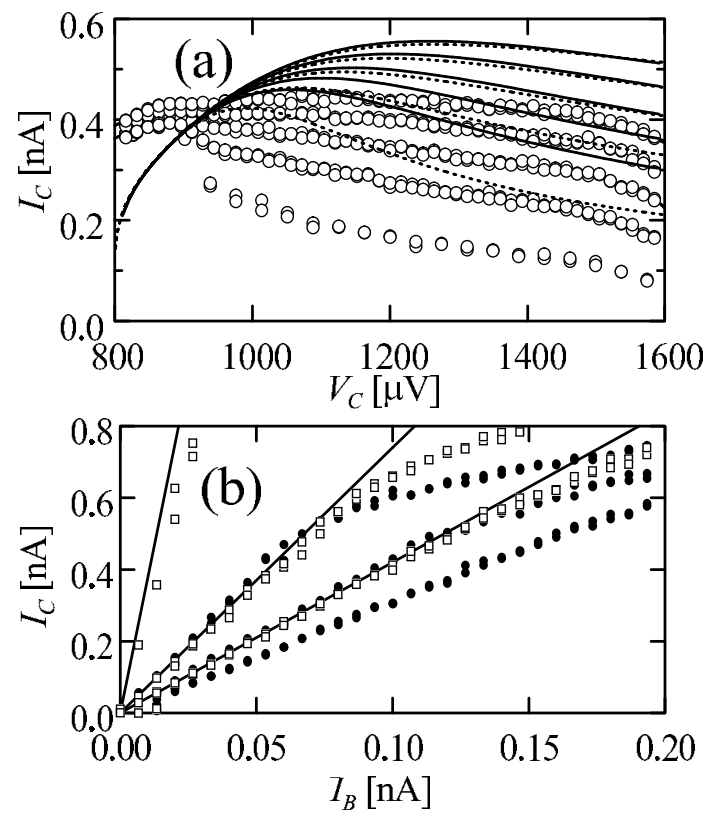

FIG. 3: (a) Computed $I_{C}-V_{C}$ plots with $R=1.5 \mathrm{M} \Omega, C=$ $0.2 \mathrm{fF}, R_{T}=12 \mathrm{M} \Omega, E_{J} / E_{C}=0.1, T=40 \mathrm{mK}$ and $\Delta=1.5$ $\mathrm{mV}$ (open circles). The base voltage has been varied as $V_{B}^{\prime}=$ $-2.5 e / C,-3.0 e / C,-3.5 e / C,-4.0 e / C,-4.5 e / C$ from down to top. Solid lines represent analytic values calculated from Eq. (9) together with approximations from Eqs. (18) and (19). Dashed lines are corrected analytic curves, which take base junction nonlinearity at the finite temperature into account. (b) Computed $I_{C}-I_{B}$ plots for the same device (solid circles) at $V_{C}=1.25 \mathrm{e} / \mathrm{C}, 1.5 \mathrm{e} / \mathrm{C}, 1.75 \mathrm{e} / \mathrm{C}$ from up to down. The open squares shows the same simulation without "Cooper pair back-tunneling" and lines show analytic predictions.

$\mathrm{M} \Omega, C=0.2 \mathrm{fF}, R_{T}=12 \mathrm{M} \Omega, E_{J} / E_{C}=0.1, T=40$ $\mathrm{mK}$ and $\Delta=1.5 \mathrm{mV}$. Corresponding analytic curves (solid lines) are calculated from Eq. (9) using the approximation of Eqs. (18) and (19) when calculating $\left\langle N_{e}\right\rangle$ and $\Gamma_{\downarrow}$. The agreement is reasonably good. An error is introduced due to the assumptions made on the shape of $\Gamma_{Q P 1}\left(V_{1}\right)$. If one uses the full temperature-dependent form (Eq. (8)) instead of Eq. (15) the agreement is improved especially at low values of $V_{B}^{\prime}$ as denoted by the dashed line in Fig. 3.

The remaining disagreement can be found to be related to the temperature dependence of Cooper pair tunneling probabilities. Even if $E_{C} / k T$ is as high as about 120 , incoherent Cooper pair tunneling enhances Cooper pair current at $V_{C} \approx V_{Q}=800 \mu \mathrm{V}$. The lower value of simulated $I_{C}$ at larger values of $V_{C}$ was found to be due to the fact that after a Cooper pair tunnels to the island it can immediately tunnel out of the island due to incoherent Cooper pair tunneling. This effectively suppresses $\langle N\rangle$, or equivalently enhances $\Gamma_{\uparrow}$. The effect is especially visible in Fig. 3(b), where a set of simulations with a current biased base electrode is performed for the same device.
The simulated curves (solid circles) fall below the theoretical curves (lines) $I_{C}=(2\langle N\rangle+1) I_{B}$ (see also Section VI), i.e. the current gain is suppressed. However, if we artificially forbid the "Cooper-pair back-tunneling" in the simulation (open squares in Fig. 3(b)) the agreement is clearly improved. This shows that the effect indeed is the main factor suppressing the current gain in the mode of operation governed by approximation given in Eqs. (18) and (19). Another mechanism due to spontaneous downwards transitions was discussed in Ref $\stackrel{4}{*}$, but it was found to be insignificant in this case.

As the tunnel resistance of the base electrode is decreased and Josephson coupling increased in simulations and experiments $\frac{3}{,}, \frac{5}{4}$ it has been found that the active bias region moves towards higher $V_{C}$ indicating that the approximation of $\left\langle N_{e}\right\rangle$ and $\Gamma_{\downarrow}$ given in Eq:s (21) and (22) becomes relevant. In Fig. 4(a) a set of simulations with parameters similar to those considered above, with exceptions $R_{T}=375 \mathrm{k} \Omega, E_{J} / E_{C}=0.2$ and $\Delta=0$ for the base junction (i.e. we have assumed that the base junction is a NIN junction here). At the upper set it is again shown a set of simulated and analytic $I_{C}-V_{C}$ curves showing a reasonable agreement. The agreement is again further improved by forbidding the "Cooper-pair back-tunneling" in the simulation, which is shown in the lower set of curves.

The devices analyzed above have relatively small $C$, which makes the voltages at which they are operated (of
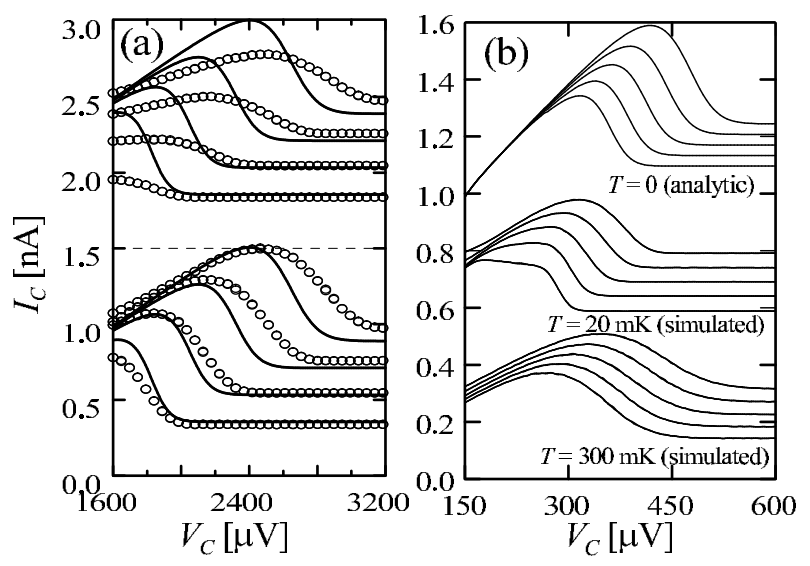

FIG. 4: (a) Computed $I_{C}-V_{C}$ plots for a device otherwise similar to that of Fig. 3 except $R_{T}=375 \mathrm{k} \Omega, E_{J} / E_{C}=0.2$ and $\Delta=0$ (an NIN base junction). The base voltages are $V_{B}^{\prime}=-1.0 e / C,-1.5 e / C,-2.0 e / C,-2.5 e / C$ from down to top (open circles). Analytic IV curves (solid lines) are calculated from (9) together with approximations from Eqs. (21) and (22). The upper set (lifted by $1.5 \mathrm{nA}$ for clarity) shows the result with the full simulation model, while the lower set shows the result without "Cooper pair back-tunneling". (b) Analytic and computed $I_{C}-V_{C}$ plots for a device having $R=500 \mathrm{k} \Omega, C=1.2 \mathrm{fF}, R_{T}=250 \mathrm{k} \Omega, \Delta=400 \mu V$ and $E_{J} / E_{C}=0.3$. The two topmost sets have been lifted by 0.5 $\mathrm{nA}$ and $0.8 \mathrm{nA}$ for clarity. 
order $V_{Q}$ ) rather high. The advantage is that the temperature dependence is minimized as $E_{C} / k T$ increases. The drawback is that higher band-gap materials are needed, since the voltage across the Josephson junction must be below $2 \Delta$. The above devices could be realized using $\mathrm{Nb}$ technology, for which $2 \Delta \approx 3 \mathrm{mV}$. More conventional Aljunctions have $2 \Delta \approx 400 \mu \mathrm{V}$, whence capacitances have to be above or around $1 \mathrm{fF}$. In Fig. 4(b) a set of $I_{C}-V_{C}$ curves are shown for a device with $R=500 \mathrm{k} \Omega, C=1.2$ $\mathrm{fF}, R_{T}=250 \mathrm{k} \Omega, E_{J} / E_{C}=0.3$. The topmost set consists of analytic curves, where at $V_{C} \lesssim 2 V_{Q} \approx 270 \mu \mathrm{V}$ approximation of Eqs. (18) and (19) and at $V_{C} \gtrsim 2 V_{Q}$ approximation of Eqs. (21) and (22) is used. The two lower sets are simulated at $T=20 \mathrm{mK}$ and $T=300 \mathrm{mK}$. Although again qualitatively similar, at $T=20 \mathrm{mK}$ the main source of disagreement is the enhancement of $\Gamma_{\uparrow}$ at a finite temperature. At $T=300 \mathrm{mK}$ the spike is spread, since at relatively large temperatures (now $E_{C} / k T \approx 2.6$ ) also $\Gamma_{\downarrow}$ is increased due to incoherent Cooper pair tunneling in a same sense as indicated in Ref $\underline{\underline{4}}$.

\section{SMALL SIGNAL PARAMETERS}

A complete small-signal model for the BOT can be given as conductance matrix

$$
\left[\begin{array}{c}
i_{C} \\
i_{B}
\end{array}\right]=\left[\begin{array}{cc}
G_{\text {out }} & g_{m} \\
g_{x} & G_{\text {in }}
\end{array}\right]\left[\begin{array}{l}
v_{C} \\
v_{B}
\end{array}\right]
$$

where $i_{C}, i_{B}, v_{C}, v_{B}$ are the small-signal components of collector and base currents and voltages, i.e. small variations around the point of operation. The definitions of small-signal parameters are listed below:

$$
\begin{aligned}
G_{\text {in }} & =\left(\frac{\partial I_{B}}{\partial V_{B}}\right)_{V_{C}} \\
g_{m} & =\left(\frac{\partial I_{C}}{\partial V_{B}}\right)_{V_{C}} \\
g_{x} & =\left(\frac{\partial I_{B}}{\partial V_{C}}\right)_{V_{B}} \\
G_{\text {out }} & =\left(\frac{\partial I_{C}}{\partial V_{C}}\right)_{V_{B}} .
\end{aligned}
$$

Note that $V_{B}$ is kept constant in the last two lines. This is the natural choice, if the circuit shown in Fig. 1(a) is used. However, if the emitter is voltage biased instead of the collector, $V_{B}^{\prime}$ should be fixed instead. The choice does not have an effect on the analysis below, since we will mostly be assuming small $R_{L}$, whence $V_{C}$ is constant. For completeness, the general formulas for small signal parameters are given here anyway.

Using Eqs. (91) and (10) we can rewrite the parameters as

$$
\begin{aligned}
G_{\text {in }} & =e\left\langle N_{e}\right\rangle\left(\frac{\Gamma_{\uparrow}}{\Gamma_{\uparrow}+\Gamma_{\downarrow}}\right)^{2} \frac{\partial \Gamma_{\downarrow}}{\partial V_{B}}\left(\beta_{B}-1\right) \\
g_{m} & =I_{S} \frac{\Gamma_{\uparrow}}{\left(\Gamma_{\uparrow}+\Gamma_{\downarrow}\right)^{2}} \frac{\partial \Gamma_{\downarrow}}{\partial V_{B}}+G_{\text {in }} \\
g_{x} & =-e\left\langle N_{e}\right\rangle \frac{\Gamma_{\downarrow} \Gamma_{\uparrow}}{\Gamma_{\uparrow}+\Gamma_{\downarrow}}\left(\frac{1}{\left\langle N_{e}\right\rangle} \frac{\partial\left\langle N_{e}\right\rangle}{\partial V_{C}}+\right. \\
& \left.+\frac{\Gamma_{\downarrow}}{\Gamma_{\uparrow}\left(\Gamma_{\uparrow}+\Gamma_{\downarrow}\right)} \frac{\partial \Gamma_{\uparrow}}{\partial V_{C}}+\frac{\Gamma_{\uparrow}}{\Gamma_{\downarrow}\left(\Gamma_{\uparrow}+\Gamma_{\downarrow}\right)} \frac{\partial \Gamma_{\downarrow}}{\partial V_{C}}\right) \\
G_{\text {out }} & =I_{S} \frac{\Gamma_{\uparrow} \Gamma_{\downarrow}}{\Gamma_{\uparrow}+\Gamma_{\downarrow}}\left(\frac{1}{\Gamma_{\downarrow}\left(\Gamma_{\uparrow}+\Gamma_{\downarrow}\right)} \frac{\partial \Gamma_{\downarrow}}{\partial V_{C}}+\right. \\
& \left.-\frac{1}{\Gamma_{\uparrow}\left(\Gamma_{\uparrow}+\Gamma_{\downarrow}\right)} \frac{\partial \Gamma_{\uparrow}}{\partial V_{C}}+\frac{1}{\Gamma_{\uparrow}} \frac{\partial I_{S}}{\partial V_{C}}\right)+g_{x},
\end{aligned}
$$

where the fact that $\Gamma_{\uparrow},\langle N\rangle$ and $I_{S}$ are independent of $V_{B}$ is used. Indices for constant quantities are dropped here for clarity. We have also defined

$$
\beta_{B}=-\frac{\Gamma_{\downarrow}\left(\Gamma_{\uparrow}+\Gamma_{\downarrow}\right)}{\Gamma_{\uparrow}\left\langle N_{e}\right\rangle}\left(\frac{\partial\left\langle N_{e}\right\rangle}{\partial V_{B}} / \frac{\partial \Gamma_{\downarrow}}{\partial V_{B}}\right)
$$

In the approximation of Eq. (18) $\beta_{B}$ is zero, since $\left\langle N_{e}\right\rangle$ is constant. Using Eqs. (21) and (22) instead makes values $\beta_{B} \approx 1$ possible. We call $\beta_{B}$ the "hysteresis parameter" of the BOT.

For some purposes it is also useful to define current gain

$$
\beta=-\left(\frac{\partial I_{C}}{\partial I_{B}}\right)_{V_{C}}=-\frac{g_{m}}{G_{i n}} .
$$

Using Eqs. (28) and (29) this is given as

$$
\beta=\frac{1}{e} \frac{I_{S}}{\Gamma_{\uparrow}\left\langle N_{e}\right\rangle\left(1-\beta_{B}\right)}+1
$$

\section{AMPLIFIER PROPERTIES}

In this section we assume that $R_{L} \ll 1 /\left|G_{\text {out }}\right|$, i.e. that BOT is read out with a current amplifier and thus $V_{C}$ is constant. For example, if one uses a dc SQUID as a postamplifier, $R_{L}$ is close to zero. Even though a voltage amplifier is used $R_{L} \approx 0$ can be realized in practice using current feedback. We will discuss two limits, one with approximation given in Eqs. (18) and (20) for evaluating $\Gamma_{\downarrow}$ and $\left\langle N_{e}\right\rangle$. In this case $\beta_{B}=0$. The second approximation uses Eqs. (21) and (22) to evaluate $\Gamma_{\downarrow}$ and $\left\langle N_{e}\right\rangle$. Then it is possible to tune $\beta_{B}$ close to unity. The emphasis is to find noise properties of the BOT at low frequencies.

The noise current at the output of the BOT is obtained by assuming that the dominant noise mechanism is the 
two-level switching noise. At low frequencies the corresponding sperctral noise density of the output current fluctuations is (see e.g. ${ }^{11}$ )

$$
S_{i, \text { out }}=4 I_{S}^{2} \frac{\Gamma_{\downarrow} \Gamma_{\uparrow}}{\left(\Gamma_{\uparrow}+\Gamma_{\downarrow}\right)^{3}},
$$

where the collector current switches between values $I_{C}=$ 0 and $I_{C}=I_{S}$. In other words we have neglected the single electron leakage from the base electrode here.

The small-signal noise model for the BOT in the limit of small $R_{L}$ is shown in Fig. 5. The signal and the noise from the source are described as current generators $i_{s g}$ and $i_{n, S}$ in parallel with the source resistance $R_{S}$. The input and output impedances are $R_{\text {in }}=1 / G_{\text {in }}$ and $R_{\text {out }}=1 / G_{\text {out }}$. The current generator $\beta i_{B}$ at the output accounts for the gain. The noise added by the BOT is represented in a standard fashion (see e.g. ${ }^{12}$ ) by equivalent voltage and current noise generators $\left(e_{n}\right.$ and $i_{n}$, respectively) at the input. According to Fig. 5 the output noise of the BOT excluding the contribution of the source $\left(i_{n, S}=0\right)$ at the output is

$$
S_{i, \text { out }}^{1 / 2}=\frac{1}{R_{\text {in }}+R_{S}} \beta S_{\text {en }}^{1 / 2}+\frac{1 / R_{\text {in }}}{1 / R_{\text {in }}+1 / R_{S}} \beta S_{\text {in }}^{1 / 2},
$$

where $S_{e n}$ and $S_{i n}$ are the spectral density functions corresponding to $e_{n}$ and $i_{n}$, respectively. Note that $e_{n}$ and $i_{n}$ and are fully correlated with equal phases. Choosing

$$
\begin{aligned}
& S_{e n}^{1 / 2}=\frac{2 I_{S}}{-g_{m}} \sqrt{\frac{\Gamma_{\downarrow} \Gamma_{\uparrow}}{\left(\Gamma_{\uparrow}+\Gamma_{\downarrow}\right)^{3}}} \\
& S_{i n}^{1 / 2}=\frac{2 I_{S}}{\beta} \sqrt{\frac{\Gamma_{\downarrow} \Gamma_{\uparrow}}{\left(\Gamma_{\uparrow}+\Gamma_{\downarrow}\right)^{3}}},
\end{aligned}
$$

the generators become independent of $R_{S}$ and produce the output noise in Eq. (35) correctly. However, the backaction noise (i.e. the noise current $i_{B A}$ through or voltage accross $R_{S}$ ) is not correctly predicted by the model.

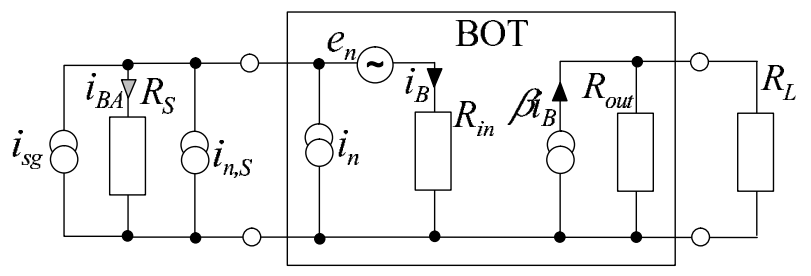

FIG. 5: A graphical representation of the small signal model of BOT in the limit of small $R_{L}$. The noise added by BOT is represented with equivalent sources $i_{n}$ end $e_{n}$.
The noise figure, defined as the ratio of total noise at the output divided by the noise contributed by the BOT, is

$$
F=1+S_{i, \text { out }}\left[\left(\frac{\beta R_{S}}{R_{\text {in }}+R_{S}}\right)^{2} S_{\text {in }, S}\right]^{-1},
$$

where $S_{i n, S}=4 k T_{0} / R_{S}$ is the spectal density function of $i_{n, S}$ and $T_{0}$ is a reference temperature. One gets optimum impedance $R_{o p t}$ and corresponding minimum noise temperature $T_{n}$ by minimizing $F$ and using the definiton $F=1+T_{n} / T_{0}$. It follows

$$
\begin{aligned}
R_{o p t} & =\sqrt{\frac{S_{e n}}{S_{i n}}}=\left|R_{i n}\right| \\
T_{n} & =\frac{1}{k_{B}} \sqrt{S_{e n} S_{i n}}=\frac{\left|R_{i n}\right| S_{i n}}{k_{B}} .
\end{aligned}
$$

The correlation of the two sources shows in Eq. (41) in such a way that the prefactor is $1 / k_{B}$ instead of $1 / 2 k_{B}$, which is the case for uncorrelated sources. The difference stems from the fact that now the amplitudes of the two sources rather than the powers are summed.

If the approximation of Eqs. (18) and (20) is used to evaluate $\left\langle N_{e}\right\rangle$ and $\Gamma_{\downarrow}$ (whence also $\beta_{B}=0$ ), one gets for gain and noise parameters

$$
\begin{aligned}
\beta & =2\langle N\rangle+1 \\
g_{m} & =-\frac{2\langle N\rangle+1}{R_{T}} \frac{1}{\left(1+\Gamma_{\downarrow} / \Gamma_{\uparrow}\right)^{2}} \\
S_{i n}^{1 / 2} & =\sqrt{-\frac{4 e}{R_{T}}\left(V_{B}^{\prime}+\frac{V_{Q}}{2}\right)\left(1+\frac{\Gamma_{\downarrow}}{\Gamma_{\uparrow}}\right)^{-3}} \\
S_{\text {en }}^{1 / 2} & =\sqrt{-4 e R_{T}\left(V_{B}^{\prime}+\frac{V_{Q}}{2}\right)\left(1+\frac{\Gamma_{\downarrow}}{\Gamma_{\uparrow}}\right)} \\
R_{o p t} & =R_{T}\left(1+\Gamma_{\downarrow} / \Gamma_{\uparrow}\right)^{2} \\
T_{n} & =-\frac{4 e}{k_{B}}\left(V_{B}^{\prime}+\frac{V_{Q}}{2}\right)\left(1+\frac{\Gamma_{\downarrow}}{\Gamma_{\uparrow}}\right)^{-1} .
\end{aligned}
$$

In this mode the BOT acts as a simple "charge multiplier", where one electron trigs $\langle N\rangle$ Cooper pairs, thus $\beta=2\langle N\rangle+1$. The current noise can also be expressed as $S_{i n}^{1 / 2}=2 \sqrt{e I_{B}}\left(1+\Gamma_{\downarrow} / \Gamma_{\uparrow}\right)^{-1}$. In the limit of small $\Gamma_{\downarrow} / \Gamma_{\uparrow}$ the Bloch oscillation sequences are short compared the total length of the "duty cycle" $1 / \Gamma_{\downarrow}+1 / \Gamma_{\uparrow}$. Then the equivalent current noise can be understood to be simply the shot noise of the input current. In that case $S_{i n}^{1 / 2}=2 \sqrt{e I_{B}}$. The prefactor 2 instead of more familiar $\sqrt{2}$ is due to the random length of charge pulses as opposed to standard shot noise. With large $\Gamma_{\downarrow} / \Gamma_{\uparrow}$, or with long Cooper pair sequences, the noise drops. The impedance also increases because single electron tunneling is forbidden during the Bloch oscillations. One should 
remember, however, that this is strictly true only in the absence of base junction leakage current.

Lowering the equivalent current noise below the input current shot noise level can be understood as follows. In the limit governed by Eqs. (42)-(46) the length of the duty cycle is determined by the base current $I_{B}$ (Eq. (10) with $\left.\left\langle N_{e}\right\rangle=1\right)$. If we have very short Cooper pair sequences (or short $1 / \Gamma_{\uparrow}$ ) compared to the duty cycle, the output current is essentially a sequence of short charge pulses of size $2 e\langle N\rangle$. This leads to shot type noise at the output, i.e. $S_{i, \text { out }}^{1 / 2}=2 \sqrt{2 e\langle N\rangle I_{C}}$ (Fig. 6(a)). The equivalent noise at the input is then $S_{i, \text { in }}^{1 / 2} \approx S_{i, \text { out }}^{1 / 2} / 2\langle N\rangle$. Using $I_{B} \approx I_{C} / 2\langle N\rangle$ we then get $S_{i, i n}^{1 / 2}=2 \sqrt{e I_{B}}$. However, if the base current is constant and the length of the Cooper pair sequence $1 / \Gamma_{\uparrow}$ (or equivalently $\langle N\rangle$ ) is increased by increasing the Josephson coupling we eventually have a situation, where $1 / \Gamma_{\downarrow}$ is the same as $1 / \Gamma_{\uparrow}$ was initially (see Fig. 6(b)). The current noise at the output is obviously the same is both cases, but the current gain $2\langle N\rangle$ is larger in Fig. 6(b). Therefore the equivalent noise at the input must be smaller.

As noted above, the spectral noise density of the backaction noise current $\left(i_{B A}\right.$ in Fig. 5) in general differs from $S_{i, i n}$. It can be shown, that for either $\Gamma_{\uparrow} / \Gamma_{\downarrow} \ll 1$ or $\Gamma_{\uparrow} / \Gamma_{\downarrow} \gg 1$, it is exactly that of the base current shot noise, i.e. $\sqrt{2 e I_{B}}$. The maximum suppression of $i_{B A}$ occurs at $\Gamma_{\uparrow}=\Gamma_{\downarrow}$, where the fano factor is $1 / 2$. The reason for the difference in the equivalent current noise and the backaction noise is, that in the limit of large $\Gamma_{\downarrow} / \Gamma_{\uparrow}$ the output current noise becomes fully anticorrelated with $i_{B A}$. Thus $i_{B A}$ does not directly determine the current resolution, or vice versa. To minimize the
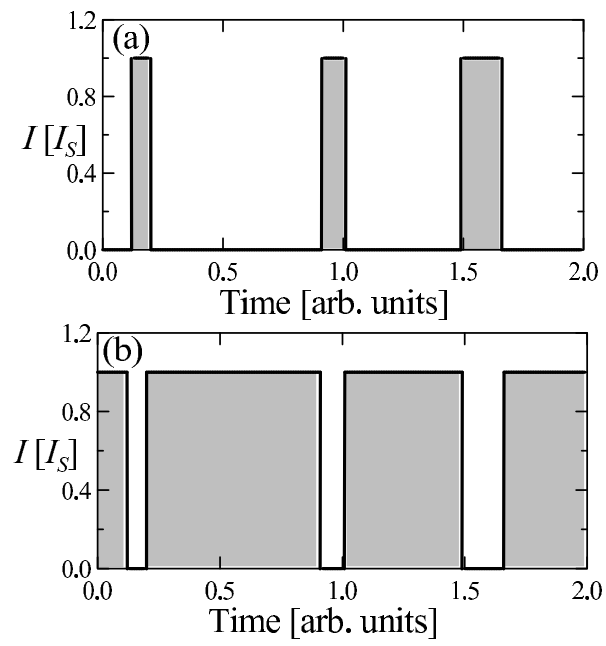

FIG. 6: Collector current $I_{C}$ schematically as function of time, when BOT is operated in the limit, where $\left\langle N_{e}\right\rangle=1$. In (a) $\Gamma_{\downarrow} / \Gamma_{\uparrow} \ll 1$ and (b) $\Gamma_{\downarrow} / \Gamma_{\uparrow} \gg 1$. The gray-shaded regions represent Cooper-pair sequences with area $2 e\langle N\rangle$. backaction noise, the device should be operated at a low base current. The low limit is here is set by spontaneous downwards transitions due to incoherent Cooper pair tunneling 4 .

To investigate the parameters from the device optimization point of view, we assume a typical point of operation, where $V_{C}=(3 / 2) V_{Q}$ and $V_{B}^{\prime}=-V_{Q}$. Then it follows

$$
\begin{aligned}
\beta & \approx 2 \exp \left(\frac{\pi R e^{2}}{8 \hbar}\left(\frac{E_{J}}{E_{C}}\right)^{2}\right) \\
g_{m} & \approx-\frac{\beta}{R_{T}} \frac{1}{\left(1+(\ln 5) / 2\left(R_{T} / R\right) \beta\right)^{2}} \\
S_{\text {in }}^{1 / 2} & \approx \sqrt{\frac{4 E_{C}}{R_{T}}\left(1+\frac{\ln 5}{2} \frac{R}{R_{T}} \beta\right)^{-3}} \\
S_{\text {en }}^{1 / 2} & \approx \sqrt{4 E_{C} R_{T}\left(1+\frac{\ln 5}{2}\left(\frac{R_{T}}{R}\right) \beta\right)} \\
R_{o p t} & \approx R_{T}\left(1+\frac{\ln (5)}{2} \frac{R}{R_{T}} \beta\right)^{2} \\
T_{n} & \approx \frac{E_{C}}{k_{B}}\left[1+\frac{\ln (5)}{2}\left(\frac{R}{R_{T}}\right) \beta\right]^{-1} .
\end{aligned}
$$

These formulas suggest, that Josephson coupling should be made large to maximize the current gain and to minimize the added noise. One should also remember that the fluctuation effects mentioned in Section IV and Ref 4 tend to suppress the gain which also increases the equivalent noise. Also the assumption $E_{J} / E_{C} \lesssim 1$ has to remain valid for the model to work.

If the approximation from Eqs. (21) and (22) is used instead of Eqs. (18) and (20) for calculating $\Gamma_{\downarrow}$ and $\left\langle N_{e}\right\rangle$, the dominating terms are in many cases those dependent on $\beta_{B}$ especially if $\beta_{B} \approx 1$. In Appendix $A$ it is presented a derivation of small signal and noise parameters. Approximations for $\Gamma_{\uparrow}, \Gamma_{\downarrow},\left\langle N_{e}\right\rangle,\langle N\rangle, \partial\left\langle N_{e}\right\rangle / \partial V_{B}$ and $\partial \Gamma_{\downarrow} / \partial V_{B}$ are made by eliminating the bias parameters at an interesting point of operation. "Fine tuning" of the device properties can be made by changing $\beta_{B}$, which in our approximation stands

$$
\beta_{B}=0.02\left(\frac{R}{R_{T}}\right)^{2} \exp \left(\frac{\pi e^{2} R}{16 \hbar}\left(\frac{E_{J}}{E_{c}}\right)^{2}\right)
$$

while other quantities of interest are 

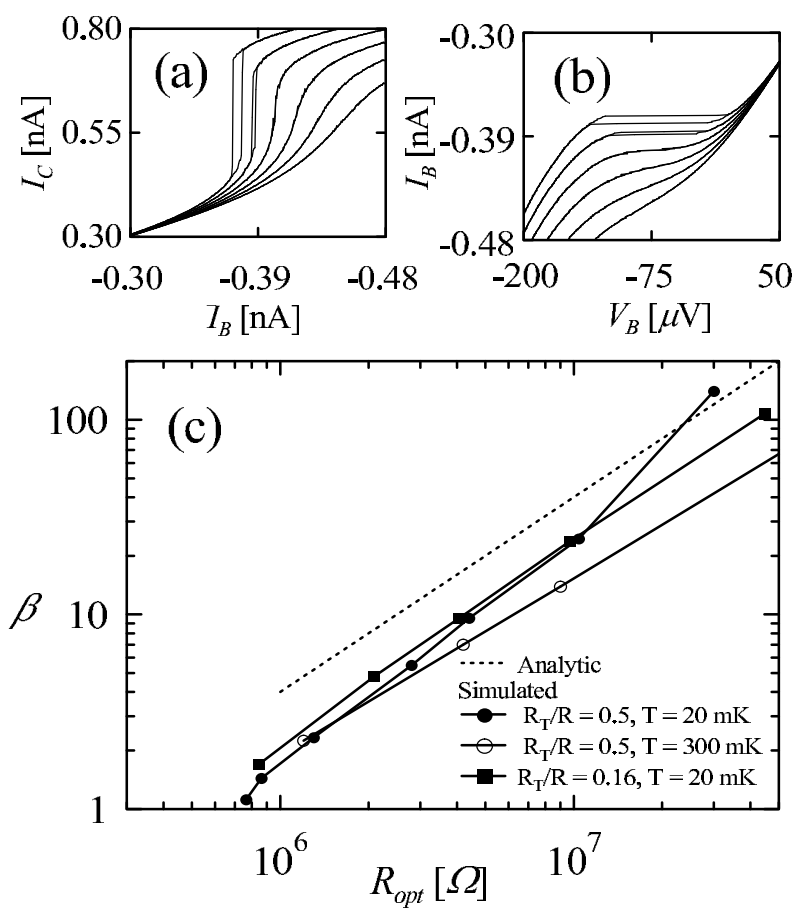

FIG. 7: (a) and (b) show a computed set $(R=500 \mathrm{k} \Omega$, $C=1.2 \mathrm{fF}, \Delta=400 \mu \mathrm{V}, T=20 \mathrm{mK}$ and $\left.R_{T} / R=0.5\right)$ of characteristic curves used to extract $\beta$ and $R_{o p t}$. The Josephson coupling is varied from $E_{J} / E_{C}=0.18$ to $E_{J} / E_{C}=0.28$ (from right to left in (a) and down to up in (b). (c) Computed and analytic current gains $\beta$ plotted against the optimum resistance $R_{o p t}$ as the Josephson coupling (or $\beta_{B}$ ) of the device is varied. The parameters are as above with the exceptions of varying $R_{T} / R$ and $T$ as shown in the legend. The bias point in the simulations with $T=20 \mathrm{mK}$ is $V_{C}=3.5 e / C$ and $V_{C}=4.5 e / C$ for those with $T=300 \mathrm{mK}$.

$$
\begin{aligned}
\beta & \approx 1.2\left(1-\beta_{B}\right)^{-1} \\
g_{m} & \approx-\frac{2}{R} \\
S_{i n}^{1 / 2} & \approx \frac{12 e}{\sqrt{R C}}\left(\frac{R_{T}}{R}\right) \beta^{-1} \\
S_{\text {en }}^{1 / 2} & \approx \frac{2 e}{\sqrt{R C}} R_{T} \\
R_{o p t} & \approx \frac{R}{2} \beta \\
T_{n} & \approx \frac{50 E_{C}}{k_{B}}\left(\frac{R_{T}}{R}\right)^{2} \beta^{-1}
\end{aligned}
$$

As $\beta_{B} \rightarrow 1$ the current gain $\beta$ diverges. However, the trade-off is that the optimum impedance $R_{o p t}$ also diverges. The fluctuation at the output does not depend on $\beta_{B}$, so the current noise $S_{i n}^{1 / 2}$ and the noise temperature $T_{n}$ decrease at the same time. The transconductance gain $g_{m}$ and voltage noise $S_{e n}^{1 / 2}$ are independent of $\beta_{B}$.
The physics in this limit can be understood as follows. With very large $\beta_{B}$ the main effect of increasing $V_{B}$ is increasing the number of electrons $\left\langle N_{e}\right\rangle$ needed to cause a downwards transition (see Eq. 32). This leads to decreasing $I_{B}$, i.e. negative input conductance. With very small $\beta_{B}$ the only effect of increasing $V_{B}$ is decreasing $\Gamma_{\downarrow}$. This leads to increasing $I_{B}$, i.e. positive input conductance ${ }^{13}$. At intermediate values, i.e. $\beta_{B} \approx 1$, the input conductance is close to zero. The effect is that a small change in $I_{B}$ causes a large change in $V_{B}$. Consequently $\Gamma_{\downarrow}$, and thus also $I_{C}$ change considerably. This leads to the enhancement of the current gain.

A set of simulated $I_{C}-I_{B}$ and $I_{B}-V_{B}$-plots with a varying Josephson coupling are shown in Figs. 7(a) and $7(\mathrm{~b})$. The parameters were chosen so that the device is realizable with $\mathrm{Al}$-tunnel junctions (see the Caption of Fig. 7). Current biased base electrode was assumed. This shows how the current gain and the input impedance increase without limit, as $\beta_{B}$ approaches unity. As $\beta_{B}$ exceeds unity the curves become hysteretic. If the source resistance $R_{S}$ is large, hysteresis is a manifestation of negative input conductance. Therefore a sufficient stability criterion for all source resistances is $\beta_{B}<1$. For small source resistances the device is stable independently of $\beta_{B}$. The simulated IV curves become hysteretic at $E_{J} / E_{C} \approx 0.25$. According to Eq. (21) $E_{J} / E_{C} \approx 0.32$ leads to $\beta_{B}=1$.

We will next illustrate the trade-offs and test the validity of Eqs. (54)-(60). The computed current gain as function of optimum resistance is shown together with the analytic approximation obtained from Eqs. (55) and (59) in Fig. 7(c). Each of the three sets have different base resistance $R_{T}$ and the bath temperature. Within each set $E_{J} / E_{C}$ is varied. One can see, that the dependence $\beta \propto R_{\text {opt }}$ is correctly reproduced regardless of parameters, i.e. the property is quite generic.

The current noise and the minimum noise temperature are shown as the function of the optimum resistance in Fig. 8. The computational noise data was obtained by performing a Fast Fourier Transform for the output current and averaging the low-frequency part, which gives $S_{i, \text { out }}$. To further evaluate $S_{i n}, R_{\text {opt }}$ and $T_{n}$, Eqs. (36), (40) and (41) were applied with computed current gain $\beta$ and input resistance $R_{i n}$. Again the correct form of dependencies, i.e. $S_{i n}^{1 / 2} \propto R_{o p t}^{-1}$ and $T_{n} \propto R_{o p t}^{-1}$ are correctly reproduced as compared to Eqs. (59) and (60). Differences in absolute levels can partially be explained through the inaccuracy of the numeric constants due to approximations made in Appendix A. To some extent the differences can also be understood with reference to excess noise mechanisms discussed in Section VII. However, correct forms of dependencies and the order of magnitude are correctly predicted with the theory. 


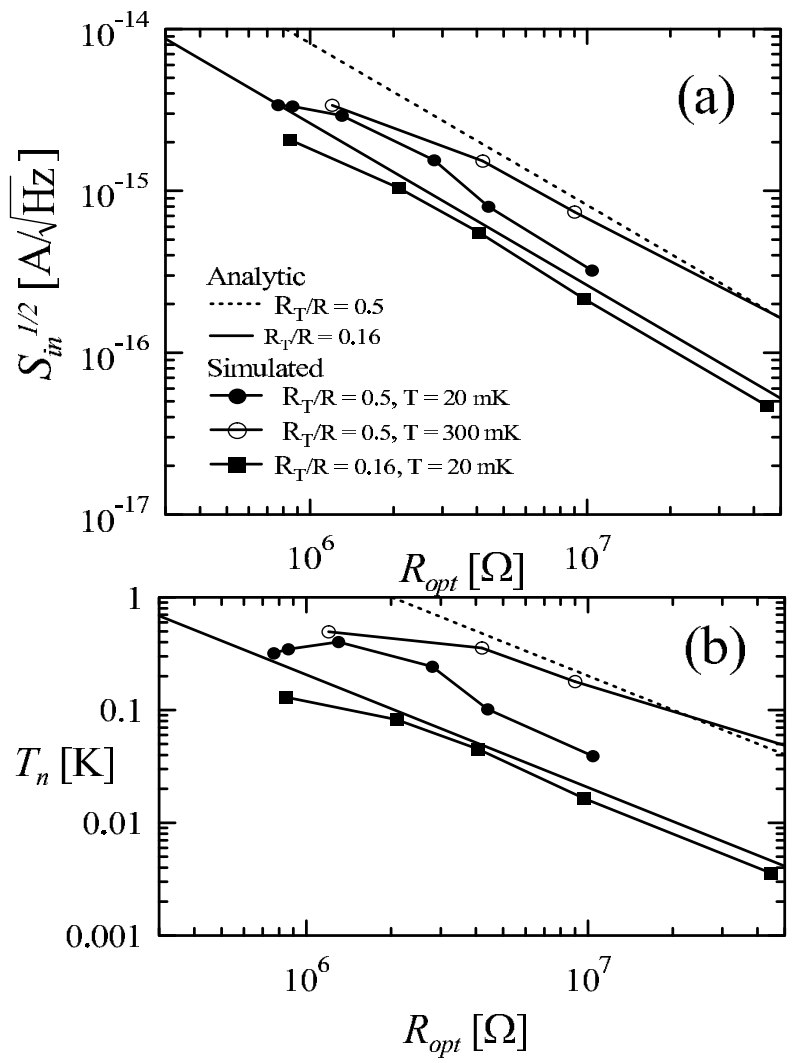

FIG. 8: (a) Computed and analytic results for the current noise spectral density $S_{i n}$ referred to input and (b) the minimum noise temperature $T_{n}$ as function of $R_{o p t}$. The device and bias parameters are the same as in Fig. 7.

\section{SUMMARY AND DISCUSSION}

We have developed an analytic theory to predict characteristic curves and noise properties of the Bloch Oscillating Transistor. Even though it was derived at zero temperature, comparison to simulations at finite temperatures showed reasonable agreement. The reason is that the main fluctuation mechanism, the two-level switching noise, is essentially temperature independent. Of other noise sources, for example, the thermal noise of the base junction is insignificant, since the junction is at a typical point of operation biased at $e V_{1} \gg k T$.

Two modes of operation were discussed. The small signal and noise parameters in both limits were obtained, i.e. Eqs. (48)-(53) and Eqs. (55)-(60). In the first mode the device acts as a simple $e-2 e\langle N\rangle$ charge multiplier. In the second mode intralevel transitions play an important role. The consequence is the emergence of the hysteresis parameter $\beta_{B}$. This was found to have a drastic effect on device properties. It was shown that noise currents below $1 \mathrm{fA}$ and noise temperatures below $100 \mathrm{mK}$ can be obtained for optimum impedances of order a few $\mathrm{M} \Omega$.

An additional noise mechanism is the shot-noise of the leakage current from the base-electrode. At a finite temperature also the bandwidth of the Bloch-oscillation increases ${ }^{6}$, and thus the shot-noise of the Cooper pairs adds to the total noise. This may explain the factor of about 3 increase in noise temperature as temperature is changed from $20 \mathrm{mK}$ to $300 \mathrm{mK}$ in Fig. 8(b). These effects appear at the output of the amplifier. Thus they add to the total output noise being additional terms to Eq. (35). Even in the presence of them the main conclusions of this article remain valid.

We have also assumed arbitrarily large $C_{B}$, which is acceptable in low-frequency applications. However, if one wishes to increase the band, $C_{B}$ should be decreased. The first effect of finite $C_{B}$ is that the base voltage starts to fluctuate at frequencies typical to BOT dynamics.

Most other well-known mesoscopic amplifiers, e.g. single-electron transistor (SET) ${ }^{14}$ or single Cooper pair transistor (SCPT) ${ }^{15}$ are based on controlling current flow by charging a gate electrode. This is similar to the field effect transistor (FET), whereas BOT resembles a bipolar junction transistor (BJT) in the sense that a small base current is used to control a larger collector current. However, there are also important differences as well. For example, we have shown that the equivalent current noise of BOT can be brought below the shot noise of the input current. The reason is, that in BOT the noise at the output is partially correlated to the noise at the input.

The $1 / f$-noise of the BOT is not addressed in this paper. However, as opposed to gate-controlled devices, the BOT is immune to background charge fluctuations. It is probable that the main $1 / f$-noise mechanism is the fluctuation of the Josephson coupling. Due to symmetry considerations, it can be reduced by using bias reversal techniques typically used with a dc SQUID (17).

Authors wish to acknowledge fruitful discussions with P. Hakonen and R. Lindell. The work was supported by the Academy of Finland (project 103948).

\section{APPENDIX A: DERIVATION OF HYSTERESIS AND NOISE PARAMETERS}

In this Appendix we show the derivation of Eqs. (54)(60). The aim is to derive an approximation, which applies at $\beta_{B} \approx 1$ and $R_{T} \lesssim R$.

The main task is to get sufficient estimates for $I_{S}, \Gamma_{\downarrow}$, $\Gamma_{\uparrow},\left\langle N_{e}\right\rangle, \partial\left\langle N_{e}\right\rangle / \partial V_{B}$ and $\partial \Gamma_{\downarrow} / \partial V_{B}$ near an interesting point of operation. We note that for the approximation from Eqs. (21) and (22) used for $\left\langle N_{e}\right\rangle$ and $\Gamma_{\downarrow}$ to apply the collector voltage must satisfy $V_{C} \gtrsim 2 V_{Q}$. For simplicity we assume that $V_{C}=2 V_{Q}$. When the system is at the second level, the voltage across the JJ is $V_{2} \gtrsim V_{Q}$ and the collector current consists of the leakage current only, i.e. $I_{C}=-I_{B}$. Assuming that $V_{2}=V_{Q}$ and that the base junction roughly acts as a linear resistor we get by analyzing the circuit of Fig. 1(a) and noting that by definition $V_{B}=V_{B}^{\prime}+V_{C}$ the result $V_{B}^{\prime}=-V_{Q}\left(1+R_{T} / R\right)$, i.e. we have found estimates for the bias parameters. Using these and Eqs. (12), (13) and (21) we can readily write: 


$$
\begin{aligned}
I_{S} & \approx \frac{2 e}{R C \ln 3} \\
\Gamma_{\uparrow} & \approx \frac{1}{R C \ln 3} \exp \left(-\frac{\pi R C}{8 \hbar} \frac{E_{J}^{2}}{E_{c}^{2}} E_{C}\right) \\
\left\langle N_{e}\right\rangle & \approx 100\left(\frac{R_{T}}{R}\right)^{2} .
\end{aligned}
$$

In the last Equation we have also utilized the assumption $R_{T} \lesssim R$.

To find an estimate for $\Gamma_{\downarrow}$ we can use physical intuition and insight learned from simulations. Since the operation is based on switching between the two states, the system spends roughly as much time in both states. Thus

$$
\Gamma_{\downarrow} \approx \Gamma_{\uparrow}
$$

Although some error may be introduced by doing this, it is not too severe, since most properties depend more strongly on the derivative $\partial \Gamma_{\downarrow} / \partial V_{B}$, which will be calculated separately.

The derivative $\partial\left\langle N_{e}\right\rangle / \partial V_{B}$ is obtained by direct differentiation of Eq. (21), inserting the bias parameters $V_{B}$, $V_{C}$ from above and applying the approximation $R_{T} \lesssim R$ :

$$
\frac{\partial\left\langle N_{e}\right\rangle}{\partial V_{B}} \approx \frac{270}{V_{Q}}\left(\frac{R_{T}}{R}\right)^{2}
$$

To obtain $\partial \Gamma_{\downarrow} / \partial V_{B}$ we first note that $\Gamma_{\downarrow}$ depends on $V_{B}$ both through the explicit dependence in Eq. 22 and through $\left\langle N_{e}\right\rangle$. Since $\left\langle N_{e}\right\rangle$ depends very strongly on $V_{B}$ we will approximate $\partial \Gamma_{\downarrow} / \partial V_{B} \approx$ $\left(\partial \Gamma_{\downarrow} / \partial\left\langle N_{e}\right\rangle\right)\left(\partial\left\langle N_{e}\right\rangle / \partial V_{B}\right)$. By differentiation of Eq. (22), application of the bias parameters $V_{B}$ and $V_{C}$ from above and using Eq. A5 we get

$$
\frac{\partial \Gamma_{\downarrow}}{\partial V_{B}} \approx 1.2 e \Gamma_{\downarrow}^{2} \frac{R+R_{T}}{V_{B}^{\prime}} \frac{\partial\left\langle N_{e}\right\rangle}{\partial V_{B}} .
$$

By using Eqs. A4 and A2 we now get

$$
\frac{\partial \Gamma_{\downarrow}}{\partial V_{B}} \approx-\frac{290}{V_{Q}} \frac{1}{R C}\left(\frac{R_{T}}{R}\right)^{2}\left(\exp \left(-\frac{\pi R C}{8 \hbar} \frac{E_{J}^{2}}{E_{c}^{2}} E_{C}\right)\right)^{2} .
$$

By inserting Eqs. (A1)-A6 into Eq. (32) we get the estimate of the hysteresis parameter $\beta_{B}$, which is shown in Eq. (54).

The exponent (or $\langle N\rangle)$ in Eqs. (A2) and A6 affects on the device parameters mainly through $\beta_{B}$. For other purposes we may assume it roughly constant and solve it by setting $\beta_{B}=1$ in Eq. (54), whence Eqs. (A2) and (A6) are simplified into

$$
\begin{gathered}
\Gamma_{\uparrow} \approx \frac{0.015}{R C}\left(\frac{R}{R_{T}}\right)^{2} \\
\frac{\partial \Gamma_{\downarrow}}{\partial V_{B}} \approx-\frac{0.08}{V_{Q}} \frac{1}{R C}\left(\frac{R}{R_{T}}\right)^{2} .
\end{gathered}
$$

Eqs. (56)- (60) are now calculated by substituting Eqs. (A1), A3), (A4), A5), (A7) and (A8) into the definitions of interesting quantities, i.e. Eqs. (29), (34), (37), (38), (40) and (41).
1 H. Seppä, and J. Hassel, cond-mat/0305263 (2003).

2 J. Hassel, and H. Seppä. IEEE Trans. Appl. Supercond 11, 260 (2001).

3 J. Delahaye, J. Hassel, R. Lindell, M. Sillanpää, M. Paalanen, H. Seppä, and P. Hakonen, Science 299, 1045 (2003).

4 J. Delahaye, J. Hassel, R. Lindell, M. Sillanpää, M. Paalanen, H. Seppä, and P. Hakonen, Phys. E 18, 15 (2003).

5 J. Hassel, J. Delahaye, H. Seppä, and P. Hakonen, cond-mat/0306023 J. Appl. Phys., in print

6 K.K. Likharev and A.B. Zorin, J. Low Temp. Phys. 59, 348 (1985).

7 G. Schön, A.D. Zaikin, Phys. Rep. 198, 237 (1990).

8 M.H. Devoret, D. Esteve, H. Grabert, G.-L. Ingold, H. Pothier and C. Urbina, Phys. Rev. Lett. 64, 1824 (1990).

9 G.-L. Ingold and Yu. V. Nazarov, in Single Charge Tunneling, edited by H. Grabert and M.H. Devoret (Plenum Press, New York 1992), pp. 21-106.
10 A.D. Zaikin and D.S. Golubev, Phys. Lett. A 164, 337 (1992).

11 Sh. Kogan, Electronic Noise and Fluctuations in Solids (Cambridge University Press 1996)

12 J. Engberg, and T. Larsen, Noise Theory of Linear and Nonlinear Circuits (John Wiley \& Sons 1995).

13 Note that with our sign conventions $\left(V_{B}<0, I_{B}<0\right)$ electrons are tunneling to the island, i.e. Increasing $V_{B}$ decreases $\Gamma_{\downarrow}$, which increases $I_{B}$. On the other hand, increasing $V_{B}$ increases $\left\langle N_{e}\right\rangle$, which decreases $I_{B}$.

14 D. Averin, and K.K Likharev, J. Low Temp. Phys. 62, 345 (1986)

15 A.B. Zorin, Phys. Rev. Lett. 76, 4408 (1996)

16 F.N.H. Robinson, Noise and Fluctuations in electronic devices and circuits, Oxford university press (1974)

17 D. Drung, Supercond. Sci. Technol. 16, 1320 (2003) 